\title{
Correlation of spontaneous adipocyte generation with osteogenic differentiation of porcine skin-derived stem cells
}

\author{
Hyo-Kyung Bae ${ }^{1}$, Bae-Dong Jung ${ }^{1}$, Seunghyung Lee ${ }^{2}$, Choon-Keun Park ${ }^{2}$, Boo-Keun Yang ${ }^{2}$, Hee-Tae Cheong ${ }^{1, *}$ \\ Colleges of ${ }^{1}$ Veterinaryy Medicine and Institute of Veterinary Science and ${ }^{2}$ Animal Life Sciences, Kangwon National University, Chuncheon 24341, Korea
}

\begin{abstract}
The objective of this study was to examine effects of spontaneous adipocyte generation on osteogenic differentiation of porcine skin-derived stem cells (pSSCs). Correlation between osteogenic differentiation and adipocyte differentiation induced by osteocyte induction culture was determined using different cell lines. Osteogenic differentiation efficiency of pSSCs was then analyzed by controlling the expression of adipocyte-specific transcription factors during osteogenic induction culture. Among four cell lines, pSSCs-II had the lowest lipid droplet level but the highest calcium content $(p<0.05)$. It also expressed significantly low levels of peroxisome proliferator-activated receptor gamma 2 (PPAR $\gamma 2$ ) and adipocyte protein $2(\mathrm{aP} 2) \mathrm{mRNAs}$ but very high levels of runt-related transcription factor 2 (Runx2) and alkaline phosphatase (ALP) mRNAs as osteogenic makers $(p<0.05)$. Oil red O extraction was increased by $0.1 \mu \mathrm{M}$ troglitazone (TGZ) treatment but decreased by $50 \mu \mathrm{M}$ bisphenol A diglycidyl ether (BADGE) $(p<0.05)$. Calcium content was drastically increased after BADGE treatment compared to that in osteogenic induction control and TGZ-treated pSSCs $(p<0.05)$. Relative expression levels of PPAR $\gamma 2$ and aP2 mRNAs were increased by TGZ but decreased by BADGE. Expression levels of Rucx2 and ALP mRNAs, osteoblast-specific marker genes, were significantly increased by BADGE treatment $(p<0.05)$. The expression level of BCL2 like 1 was significantly higher in BADGE-treated pSSCs than that in TGZ-treated ones $(p<0.05)$. The results demonstrate that spontaneous adipocyte generation does not adversely affect osteogenic differentiation. However, reducing spontaneous adipocyte generation by inhibiting PPAR 2 mRNA expression can enhance in vitro osteogenic differentiation of pSSCs.
\end{abstract}

Keywords: Porcine skin-derived stem cells; Osteogenesis; Spontaneous adipocyte generation; Peroxisome proliferator-activated receptor gamma 2

\section{Introduction}

The biochemical environment of bone is composed of connective tissue, nerve, tendon, and fat tissue. The process of differentiation into a specific cell type can be influenced by the native environment of bone. Bone adipocyte numbers in bone marrow are modified under various biological conditions [20]. Adipocytes and osteoblasts are differentiated from mesenchymal stem cells (MSCs). These cells co-exist in bone marrow. Because both cells are derived from MSCs, osteogenic capability might decrease while the differentiation potential of cells is being consumed to differentiate into adipocytes [4]. Although many in vitro and in vivo studies have discussed adipose tissue-produced factors related to osteogenesis $[8,18]$, the correlation of adipocyte existence with bone cells during osteogenesis remains unclear.

Recently, we reported on differentiation induction of porcine skin-derived stem cells (pSSCs) into three mesodermal cell lineages in vitro [10]. In previous studies, lipid (such as free fatty acids) droplets have been generated not only during adipogenic differentiation but also during chondrogenic differentiation [3] and osteogenic differentiation of pSSCs (data not shown). Under a defined culture condition, osteogenic determination is controlled by various cytokines, growth factors, and specific transcription regulators such as peroxisome proliferator-activated receptor gamma (PPAR $\gamma)$, runt-related transcription factor 2 (Runx2), alkaline phosphatase (ALP), collagen type I (Col I), osteonectin, and osteorix [11,32]. Especially, PPAR $\gamma$ has an essential role in regulating differentiation into both adipocytes and osteoblasts [31]. 
It has been shown that stimulation of PPAR $\gamma$ by an agonist can drastically enhance the adipogenic differentiation of porcine stromal-vascular cells [24]. Thiazolidinediones (TZD), selective PPAR $\gamma$ receptor agonists, have been used for research on bone mineral density and in treatments for hyperlipidemia and type 2 diabetes by improving sensitivity of cells to insulin [2,14]. TZD such as rosiglitazone (RGZ), troglitazone (TGZ), and pioglitazone (PGZ) are potent PPAR $\gamma$ agonists that can stimulate adipogenesis of MSCs [16]. Previous studies have shown that treatment with TGZ can promote adipogenic differentiation and proliferation and increase the number of small adipocytes via activation or expression of PPAR $\gamma$ in porcine [19]. Bisphenol A diglycidyl ether (BADGE), another PPAR $\gamma$ regulator, is a low-affinity PPAR $\gamma$ ligand with PPAR $\gamma$ antagonist features that can block adipocyte differentiation [5]. BADGE, a PPAR $\gamma$ antagonist, is reported to be able to block adipocyte differentiation in mice and prevent binding of PPAR $\gamma$ agonists and PPAR $\gamma$ transcriptional activity in 3T3-L1 cells [5].

The purpose of this study was to examine whether spontaneous adipocyte generation could affect osteogenic differentiation of pSSCs. Correlation between osteogenic differentiation and adipocytes differentiation induced by osteocyte induction culture was first determined in different cell lines. In addition, osteogenic differentiation efficiency of pSSCs was analyzed by regulating the expression of adipocyte-specific transcription factors by TGZ or BADGE treatment during osteogenic induction culture.

\section{Materials and Methods}

\section{Cell isolation}

Porcine skin samples were obtained from ear tissues of pigs (6-month-old females, $n=4)$. First, ear tissues were placed in Dulbecco's phosphate-buffered saline (DPBS; WELGENE, Korea) with $2 \%$ penicillin/streptomycin (P/S; Corning Cellgro, USA). To isolate pSSCs, cartilage tissue was completely removed from the ear skin sample. Epidermis and dermis of the skin tissue were then washed twice with warmed Hank's balanced salt solution (WELGENE). These specimens were finely chopped with a scalpel blade and treated with a digestion solution containing $0.25 \%$ trypsin-EDTA (Sigma-Aldrich, USA) and $0.1 \%$ collagenase type I in DPBS for $30 \mathrm{~min}$ at $37^{\circ} \mathrm{C}$ with agitation. The pSSCs were recovered and plated into 100 mm culture dishes (BD Biosciences, USA) and cultured in Dulbecco's modified Eagle's medium (DMEM; Gibco, USA) supplemented with $10 \%$ heat-inactivated fetal bovine serum (FBS; Gibco) and 1\% P/S in humidified air with 5\% (v/v) $\mathrm{CO}_{2}$ at $37^{\circ} \mathrm{C}$ for more than 10 days. Cells from each pig were cultured separately and passaged once or twice with culture medium changed every two days. MSC characteristics of pSSCs were confirmed by fluorescence-activated cell sorting analysis as described previously [10].

\section{Osteogenic differentiation in vitro}

To induce osteogenesis, pSSCs were plated in 24-well plates at a density of $2 \times 10^{4}$ cells $/ \mathrm{cm}^{2}$ and cultured in osteogenic induction medium consisting of $10 \mathrm{mM}$ glycerol 2-phosphate (Sigma-Aldrich), $50 \mu \mathrm{M}$ ascorbic acid, $0.1 \mu \mathrm{M}$ dexamethasone, $1 \%$ antibiotics, and $10 \%$ FBS. The medium was changed every 2 to 3 days. Non-induced and osteogenic-induced cells were fixed in 4\% paraformaldehyde for $20 \mathrm{~min}$. Mineralized cells were stained for calcium deposition with von Kossa stain. These fixed cells were washed three times with deionized water, treated with 5\% silver nitrate (Sigma-Aldrich), and exposed to strong light for $1 \mathrm{~h}$. The reaction was stopped by treatment with $5 \%$ sodium thiosulfate nitrate (Sigma-Aldrich) for 2 to $3 \mathrm{~min}$ and the cells examined under a light microscope (TE300; Nikon, Japan). Finally, the amount of calcium deposited in each group was determined by using a calcium assay kit (QuantiChrom Calcium Assay Kit [DICA-500]; Bioassay System, USA). Optical density (OD) of the extracted dye was measured at a wavelength of $612 \mathrm{~nm}$ using an enzyme-linked immunosorbent assay (ELISA) plate reader (VersaMax; Molecular Devices, USA).

\section{PPAR $\gamma$ regulator treatment}

For treatment with TGZ and BADGE as PPAR $\gamma$ regulators, pSSCs were cultured in osteogenic induction medium with either TGZ (TOCRIS, USA) at $0.01,0.1$, and $1 \mu \mathrm{M}$ or BADGE (TOCRIS) at $10,20,50$, and $100 \mu \mathrm{M}$ for 24 days at $37^{\circ} \mathrm{C}$ in humidified air with $5 \%(\mathrm{v} / \mathrm{v}) \mathrm{CO}_{2}$. The medium was changed every two days. Stock solutions of TGZ and BADGE were prepared in dimethyl sulfoxide (DMSO; Junsei Chemical, Japan). Non-induced pSSCs cultured for 24 days without osteogenic induction factors were used as the negative control.

\section{Oil red $\mathrm{O}(\mathrm{ORO})$ staining and extraction quantification}

For analysis of adipocytes generated during osteogenic differentiation, ORO staining and quantification were conducted as described previously [10]. Briefly, pSSCs were stained with a $0.6 \%$ ORO (Sigma-Aldrich) solution (w/v) for $1 \mathrm{~h}$ at room temperature. Stained cells were examined under a light microscope (TE300). The ORO was eluted with isopropanol and quantified by measuring OD at a wavelength of $510 \mathrm{~nm}$ with an ELISA plate reader.

\section{RNA preparation and quantitative real-time polymerase chain reaction (qRT-PCR) analysis}

Total RNA was extracted from induced cells using Trizol (Invitrogen, Germany) and following the manufacturer's instructions in order to evaluate gene expression of osteoblast and adipocyte markers. To carry out PCR, total RNA was diluted to $500 \mathrm{ng} / \mu \mathrm{L}$ using RNase-free water and reverse transcribed into cDNA using RT-PreMix (Bioneer, Korea). The cDNA synthesis was carried out in a Veriti 96-well Thermal 
cycler (Applied Biosystems, USA) using the following schedule: $37^{\circ} \mathrm{C}$ for $15 \mathrm{sec}, 50^{\circ} \mathrm{C}$ for $4 \mathrm{~min}$, and $60^{\circ} \mathrm{C}$ for $30 \mathrm{sec}$. The cDNA was then diluted 1:5 (v/v) with RNase-free water prior to qRT-PCR which was performed using $1 \mu \mathrm{L}$ of diluted cDNA in combination with power SYBR Green PCR master Mix (TOPreal qPCR 2X PreMIX [SYBR Green with high ROX]; Enzynomics, Korea). The amplification reaction was conducted in a StepOnePlus instrument (Applied Biosystems) with the following schedule: 40 cycles of denaturation at $95^{\circ} \mathrm{C}$ for $30 \mathrm{sec}$, annealing at $60^{\circ} \mathrm{C}$ for $30 \mathrm{sec}$, and extraction at $72^{\circ} \mathrm{C}$ for $30 \mathrm{sec}$. All quantitative data were analyzed by applying the comparative $\Delta \Delta^{\mathrm{CT}}$ method after normalization against the expression level of the corresponding housekeeping gene glyceraldehyde-3-phosphate dehydrogenase (GAPDH) and the non-induction group to obtain relative fold-change values.
Experiments for each sample were conducted in triplicates. Primers used for amplification of PPAR $\gamma 2$, adipocyte protein 2 (aP2), CCAAT/enhancer-binding protein-alpha $(\mathrm{C} / \mathrm{EBP}-\alpha)$, runt-Runx2, ALP, osteopontin (SPP1), Col I, BCL2 like 1 (BCL2L1), BCL2-associated X protein (BAX), caspase-3 (Casp-3), insulin-like growth factor 1 (IGF1), IGF1-receptor (IGF1-R), $\beta$-catenin, and GAPDH are shown in Table 1.

\section{Statistical analysis}

Results are expressed as mean \pm SE values from triplicate experiments. Cells from each group were evaluated separately. Statistical significance was determined by analysis of variance and Duncan's multiple range test included in the Statistical Analysis System software package (SAS Institute, USA).

Table 1. Sequences of primers used for quantitative real-time polymerase chain reaction

\begin{tabular}{|c|c|c|c|c|}
\hline Gene & Primer sequence $\left(5^{\prime}-3^{\prime}\right)$ & Size (bp) & $\begin{array}{c}\text { GenBank } \\
\text { accession No. }\end{array}$ & $\begin{array}{c}\text { Annealing } \\
\text { temperature }\left({ }^{\circ} \mathrm{C}\right) \text {, } \\
(40 \text { cycle })\end{array}$ \\
\hline \multirow[t]{2}{*}{ PPAR $\gamma 2$} & F-GCGCCCTGGCAAAGCACT & 238 & AF103946 & 62 \\
\hline & R-TCCACGGAGCGAAACTGACAC & & & \\
\hline \multirow[t]{2}{*}{ aP2 } & F-GGCCAAACCCAACCTGA & 167 & AF102872 & 62 \\
\hline & R-GGGCGCCTCCATCTAAG & & & \\
\hline \multirow[t]{2}{*}{ C/EBP- $\alpha$} & F-CTGGAGCTGACCAGTGACAA & 209 & AF103944 & 62 \\
\hline & R-AGATCTGGAGACCCGAAACC & & & \\
\hline \multirow[t]{2}{*}{ Runx2 } & F-CAGACCAGCAGCACTCCATA & 171 & EU668154 & 60 \\
\hline & R-AACGCCATCGTTCTGGTTAG & & & \\
\hline \multirow[t]{2}{*}{ Col I } & F-CCAAGAGGAGGGCCAAGAAGAAGG & 232 & AF201723 & 62 \\
\hline & R-GGGGCAGACGGGGCAGCACTC & & & \\
\hline \multirow[t]{2}{*}{ ALP } & F-CCACACCGTGGACTACCTCT & 152 & AY145131 & 62 \\
\hline & R-CCAGCAAGAAGAAGCCTTTG & & & \\
\hline \multirow[t]{2}{*}{ SPP1 } & F-CTTGGACAGCCAAGAGAAGG & 165 & NM214023 & 60 \\
\hline & R-TGGCTGACTTTGGGATTTTC & & & \\
\hline \multirow[t]{2}{*}{ BCL2L1 } & F-GTTGACTTTCTCTССТACAAGC & 277 & NM_214285.1 & 55 \\
\hline & R-GGTACCTCAGTTCAAACTCATC & & & \\
\hline \multirow[t]{2}{*}{ BAX } & F-ACTGGACAGTAACATGGAGC & 294 & XM003127290.3 & 55 \\
\hline & R-GTCCCAAAGTAGGAGAGGAG & & & \\
\hline \multirow[t]{2}{*}{ Casp-3 } & F-GAGGCAGACTTCTTGTATGC & 237 & NM_214131 & 60 \\
\hline & R-CATGGACACAATACATGGAA & & & \\
\hline \multirow[t]{2}{*}{ IGF1 } & F-ATTTCTTGAAGGTAAAGATGCA & 117 & DQ784687 & 60 \\
\hline & R-CAGCCCCACAGAGGGTCTCA & & & \\
\hline \multirow[t]{2}{*}{ IGF1-R } & F-CGAGAGACATCTATGAGACA & 382 & AB003362 & 57 \\
\hline & R-TCCTCACTGTAGTAGAAGGA & & & \\
\hline \multirow[t]{2}{*}{$\beta$-catenin } & F-TGCCACACGTGCAATCCCCG & 396 & NM_214367.1 & 60 \\
\hline & R-TGCAGCCCGCCAGCTAAACG & & & \\
\hline \multirow[t]{2}{*}{ GAPDH } & F-GGGCATGAACCATGAGAAGT & 230 & AF017079 & 60 \\
\hline & R-AAGCAGGGATGATGTTCTGG & & & \\
\hline
\end{tabular}

PPAR 2 2, peroxisome proliferator-activated receptor gamma 2; aP2, adipocyte protein 2; C/EBP- $\alpha$, CCAAT/enhancer-binding protein-alpha; Runx2, runt-related transcription factor 2; Col I, collagen type I; ALP, alkaline phosphatase; SPP1, osteopontin; BCL2L1, BCL2 like 1; BAX, BCL2-associated X protein; Casp-3, caspase-3; IGF1, insulin-like growth factor 1; IGF1-R, IGF1-receptor; F, forward; R, reverse. 


\section{Results}

\section{Osteogenic differentiation potential of various pSSC lines}

Following osteogenic induction for 24 days, all four pSSC lines derived from different individuals (pSSCs-I, -II, -III, and -IV) showed not only adipocyte formation but also nodule formation and aggregation in clusters, which are morphological features of osteoblastic cells. However, concentrations of lipid droplets varied among these cell lines, with pSSCs-IV having a higher concentration of lipid droplets than that in the other cell lines (panel A in Fig. 1).

Quantitative analysis of ORO and von Kossa staining results revealed that levels of lipid droplet and calcium contents were significantly higher in induced pSSC lines than those in corresponding non-induced controls $(p<0.05)$ (panels B and $\mathrm{D}$ in Fig. 1). Among the four cell lines, pSSCs-IV had the highest level of lipid droplets (OD, $6.70 \pm 0.13 ; p<0.05)$ whereas pSSCs-II had the lowest level (OD, $1.89 \pm 0.10)$ (panel $\mathrm{C}$ in Fig. 1). In contrast, calcium content was higher in pSSCs-II $(\mathrm{OD}, 3.39 \pm 0.17 ; p<0.05)$ than in the others (OD: pSSCs-I, $2.00 \pm 0.02$; pSSCs-III, $1.72 \pm 0.04 ;$ pSSCs-IV, $1.92 \pm 0.05$ ) (panel E in Fig. 1).
A psscs-l
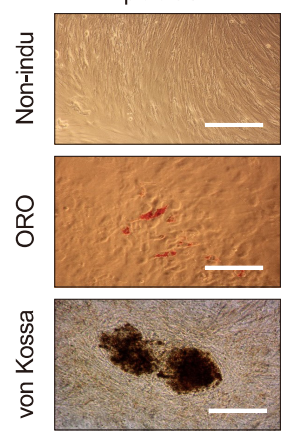

乌े

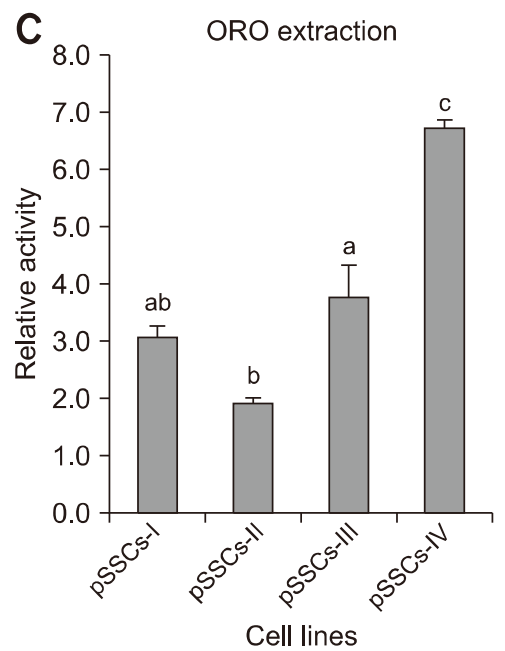

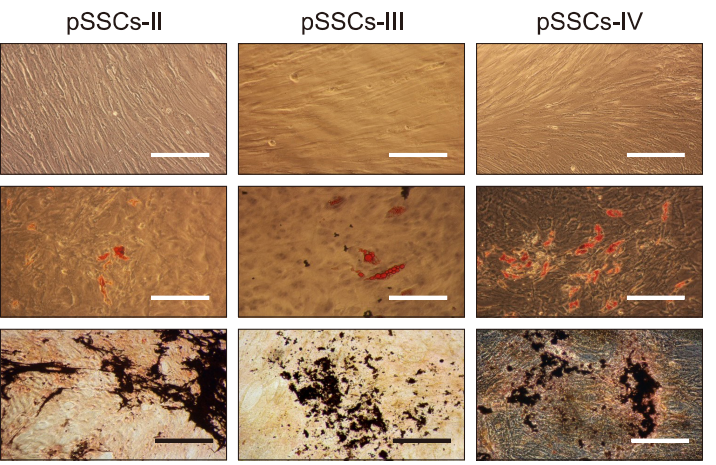
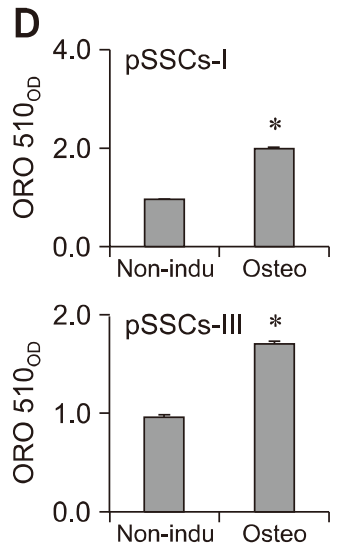

B
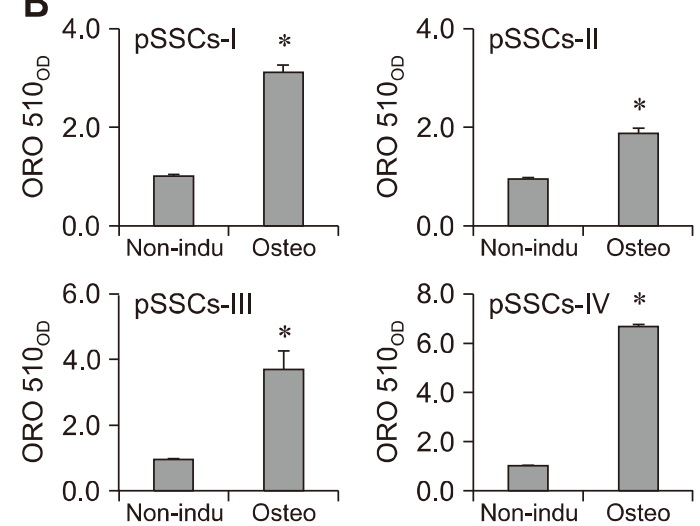

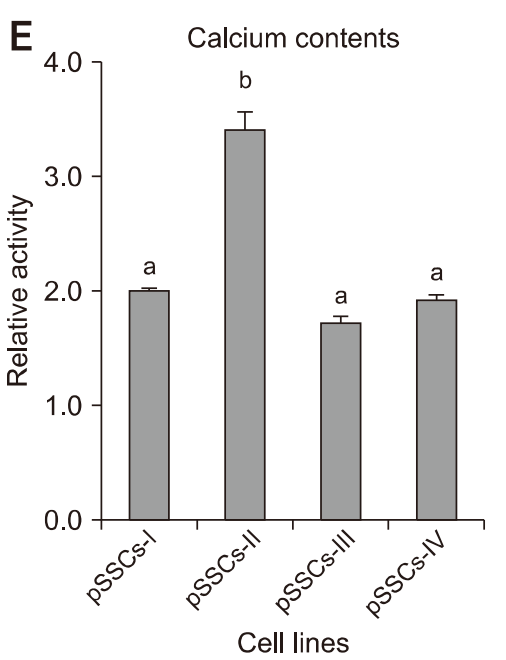

Fig. 1. Analysis of osteogenic differentiation in porcine skin-derived stem cells (pSSCs). (A) Morphological images of osteogenic-induced pSSCs from four individual cell lines (pSSCs-I, -II, -III, and -IV). After induction for 24 days, spontaneous lipid droplets generated during osteogenic differentiation of pSSCs were stained by Oil red O (ORO; red, positive cells). Osteogenesis was assessed by von Kossa staining for mineralization in induced cells. Control cells (Non-indu) were cultured in Dulbecco's modified Eagle's medium $+10 \%$ fetal bovine serum for the same duration. Scale bars $=100 \mu \mathrm{m}(\mathrm{A})$. (B-E) Quantitative data of lipid droplet formation and osteogenic differentiation potentials from pSSCs-I, -II, -III, and -IV. Levels of lipid droplets and calcium contents in osteogenic-induced pSSCs after 24 days of osteogenic differentiation were determined by ORO and calcium deposit results, respectively. (B and C) Quantitative data based on ORO staining. Data in panel C in Fig. 1 are represented as fold-change from non-induced control cells. ( $D$ and E) Quantitative data of calcium contents. Data in panel E in Fig. 1 are represented as fold-change from non-induced control cells. OD, optical density; Non-indu, non-induction; Osteo, osteogenic induction. *Significantly higher than non-induction control $(p<0.05) .{ }^{\mathrm{a}-\mathrm{C}}$ Values with different letters differ significantly $(p<0.05)$. 


\section{Expression patterns of adipogenic and osteogenic markers in pSSC lines}

Results of qRT-PCR analysis of osteogenic-induced pSSCs revealed that the expression patterns of three adipogenic markers varied: expression levels of PPAR $\gamma 2$ and aP2 mRNAs were very high in pSSCs-IV but very low in pSSCs-II $(p<$ 0.05 ), whereas the C/EBP- $\alpha$ mRNA level was high in pSSCs-III (Fig. 2). Expression patterns of osteogenic makers also varied by cell line. Levels of Runx 2 and ALP mRNAs were relatively high in pSSCs-II but significantly low in pSSCs-IV $(p<0.05)$. In contrast, expression levels of Col I and SPP1 mRNAs were relatively low in pSSCs-II but high in pSSCs-I and pSSCs-IV.

\section{Osteogenic differentiation potential of pSSCs following treatment with TGZ and BADGE}

Whether adipocyte formation could alter osteogenic differentiation in comparison with PPAR $\gamma$ regulators was then examined. To examine the effect of various concentrations of TGZ and BADGE on expression of PPAR $\gamma 2$ mRNA, a key transcription factor of adipogenesis, pSSCs were cultured in osteogenic induction medium with either TGZ $(0.01,0.1$, or 1 $\mu \mathrm{M})$ or BADGE $(10,20,50$, or $100 \mu \mathrm{M})$ for 24 days. Results showed that the PPAR $\gamma 2$ mRNA expression level in osteogenic-induced cells was significantly higher in $0.1 \mu \mathrm{M}$ TGZ-treated pSSCs than that in osteogenic-induced control or other TGZ treatment groups $(p<0.05)$ (panel A in Fig. 3). After treatment with various concentrations of BADGE, the PPAR $\gamma 2$ mRNA expression level decreased with increasing concentration of BADGE. It was markedly decreased following
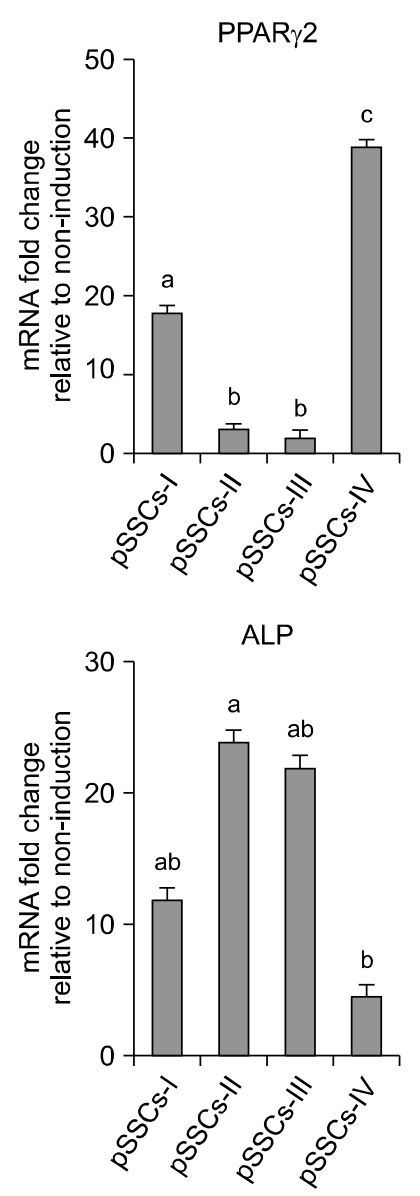

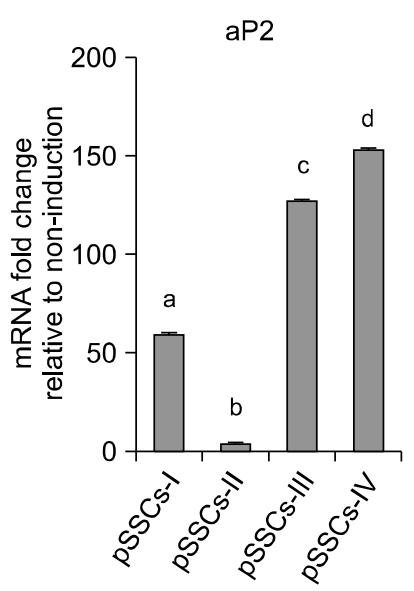

Col I

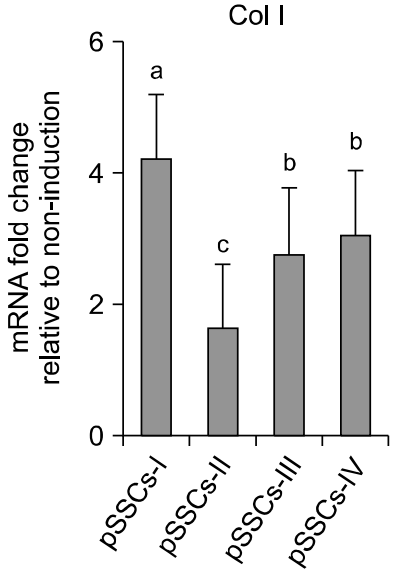

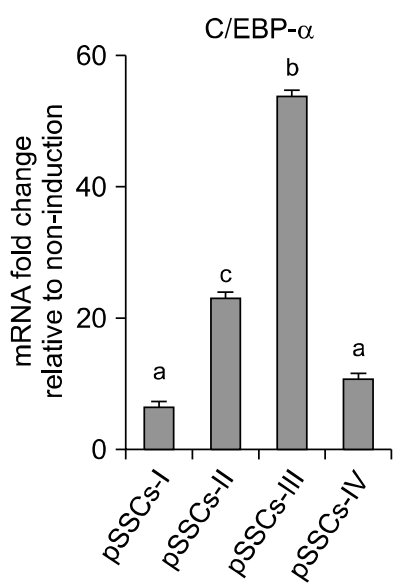
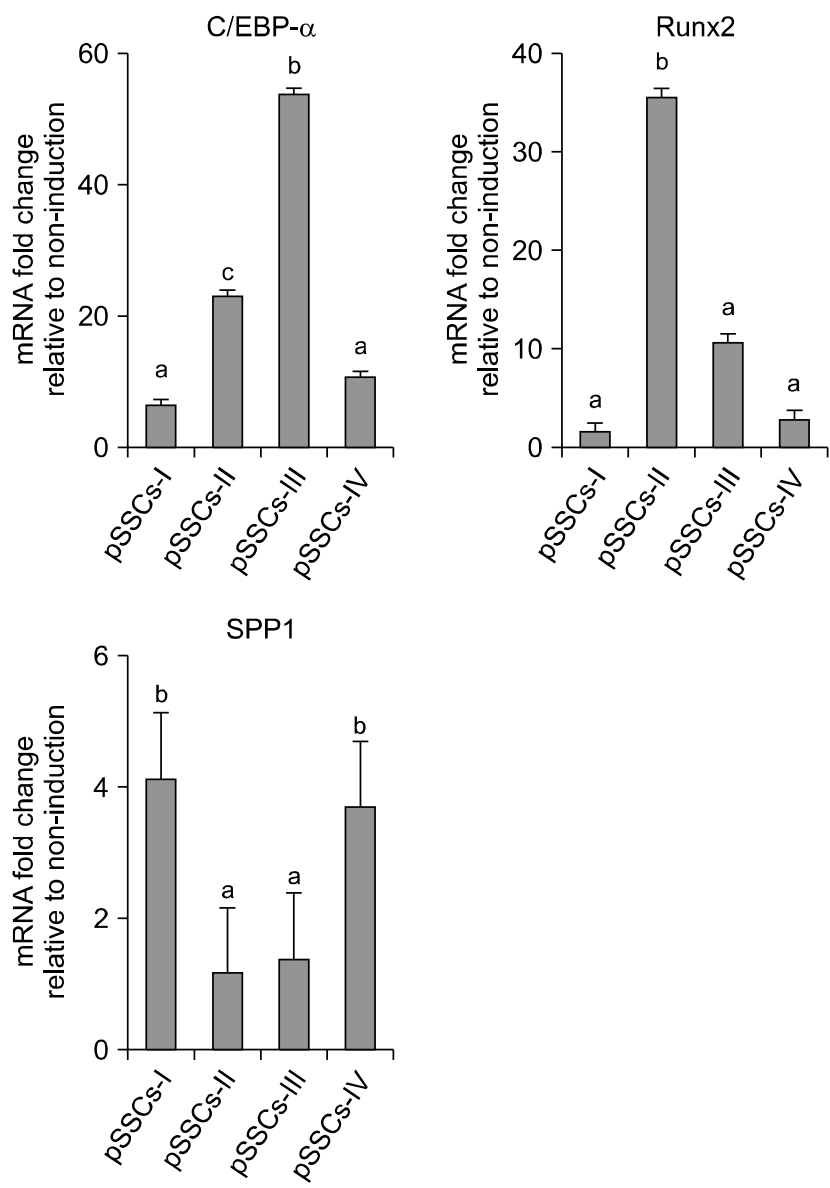

Fig. 2. Gene expression levels of osteoblast and adipocyte markers in four osteogenic-induced porcine skin-derived stem cell (pSSC) lines. Gene expression levels were analyzed by quantitative real-time polymerase chain reaction, normalized to glyceraldehyde-3-phosphate dehydrogenase (GAPDH), and represented as fold-change from non-induced control cells. Data (mean \pm SE) of pSSCs-I, -II, -III, and -IV were obtained from four donor biological samples with at least three trials. PPAR 2 , peroxisome proliferator-activated receptor gamma 2; aP2, adipocyte protein 2; C/EBP- $\alpha$, CCAAT/enhancer-binding protein-alpha; Runx2, runt-related transcription factor 2; ALP, alkaline phosphatase; Col I, collagen type I; SPP1, osteopontin. ${ }^{\text {a-d }}$ Values with different letters differ significantly ( $p<0.05)$. 

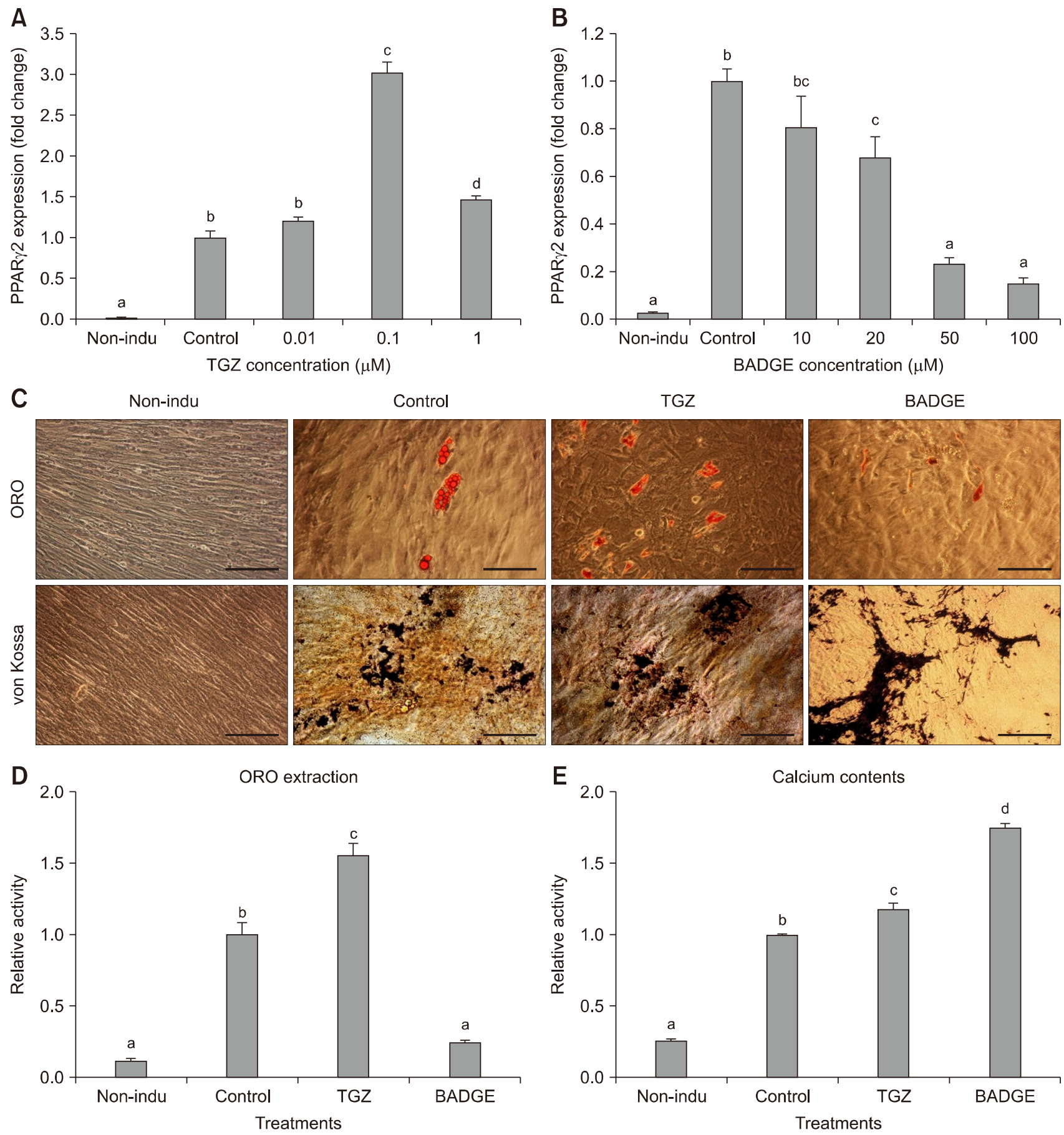

Fig. 3. Osteogenic differentiation potential changes of porcine skin-derived stem cells (pSSCs) treated by peroxisome proliferator-activated receptor gamma (PPAR $\gamma$ ) regulators. Detection of lipid droplets and calcium deposition in osteogenic induction culture of pSSCs following treatment with troglitazone (TGZ) or bisphenol A diglycidyl ether (BADGE). (A and B) PPAR $\gamma 2$ (PPAR $\gamma 2$ ) mRNA expression level following treatment with various concentrations of TGZ and BADGE during osteogenic differentiation. (C) Oil red $\mathrm{O}(\mathrm{ORO})$ and von Kossa stained images. Scale bars $=100 \mu \mathrm{m}(\mathrm{C})$. (D) Relative ORO extraction level of osteogenic-induced pSSCs. (E) Relative calcium contents of osteogenic-induced pSSCs. These pSSCs were cultured in osteogenic medium with $0.1 \mu \mathrm{M}$ TGZ or 50 $\mu \mathrm{M}$ BADGE for 24 days. Data are expressed as relative activity compared to osteogenic-induced control (mean $\pm \mathrm{SE}$ ) from three replicates. Non-indu, non-induction. ${ }^{a-d}$ Values with different letters differ significantly $(p<0.05)$. 
$50 \mu \mathrm{M}$ BADGE treatment (panel B in Fig. 3). Thus, $0.1 \mu \mathrm{M}$ TGZ and $50 \mu \mathrm{M}$ BADGE were used for subsequent experiments.

Generation of lipid droplets (adipocytes) and mineralized bone structures after induction culture were confirmed by ORO and von Kossa staining, respectively (panel C in Fig. 3). Treatment with the PPAR $\gamma$ agonist TGZ increased the number of lipid droplets, but the size and number of lipid droplets decreased in BADGE-treated cells (panel $\mathrm{C}$ in Fig. 3). ORO extraction was increased by TGZ treatment but decreased by BADGE treatment (OD: induction control, $1.00 \pm 0.09$; TGZ, $1.55 \pm 0.09$; BADGE, $0.24 \pm 0.02 ; p<0.05$ ) (panel D in Fig. 3). On the other hand, calcium content was significantly increased in both TGZ- and BADGE-treated pSSCs, whereas a remarkable increase of calcium content was only obtained after BADGE treatment compared to that in the osteogenic induction control and TGZ-treated pSSCs (OD: induction control, $1.00 \pm$ 0.00 ; TGZ, $1.18 \pm 0.05$; BADGE, $1.74 \pm 0.03 ; p<0.05$ ) (panel E in Fig. 3).

\section{Expression patterns of adipogenic and osteogenic markers in pSSCs following treatment with TGZ and BADGE}

Expression levels of mRNAs associated with adipocyte and osteoblast in osteogenic-induced pSSCs treated with $0.1 \mu \mathrm{M}$ TGZ and $50 \mu \mathrm{M}$ BADGE for 24 days were assessed by qRT-PCR. In the induced control groups, genes related to adipocyte and osteoblast were expressed (Fig. 4). Relative expression level of PPAR $\gamma 2$ mRNA was increased by TGZ
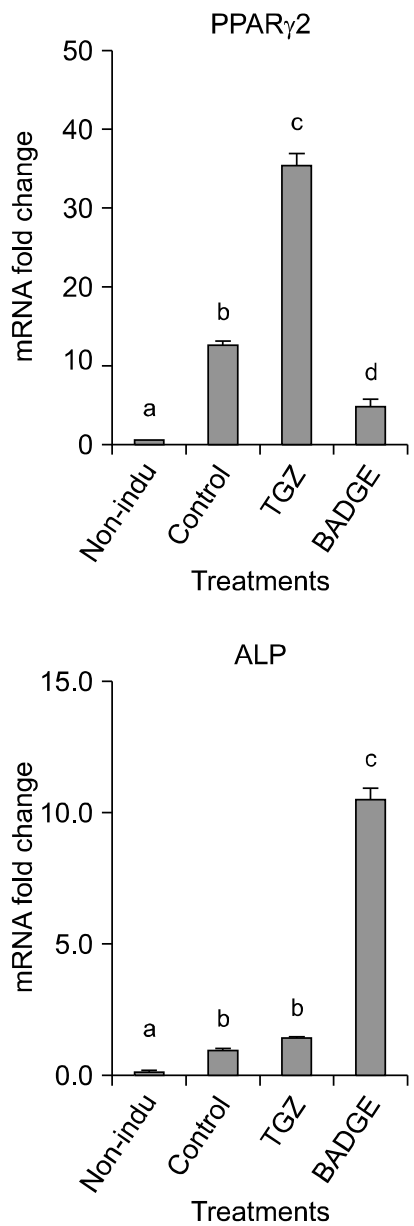

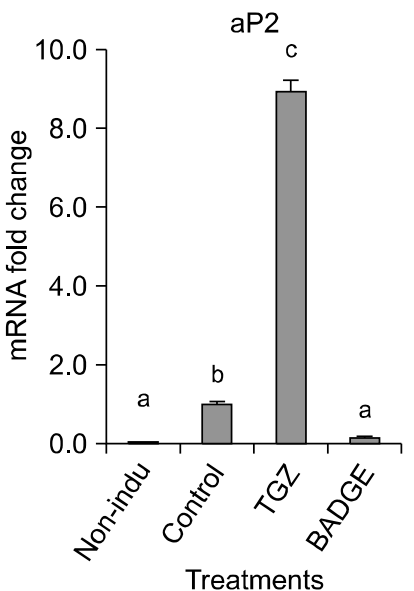

SPP1

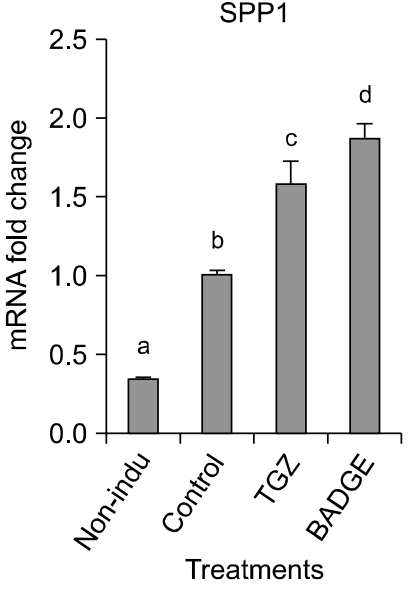

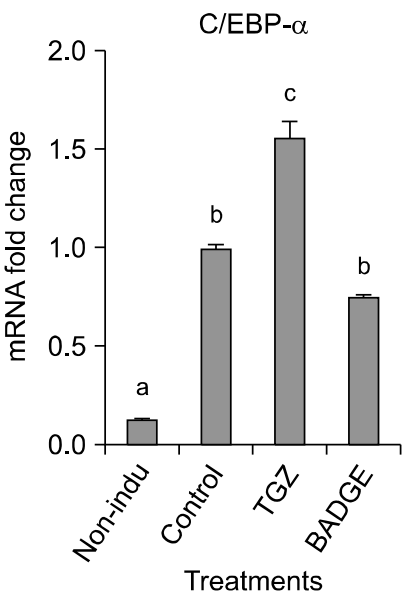

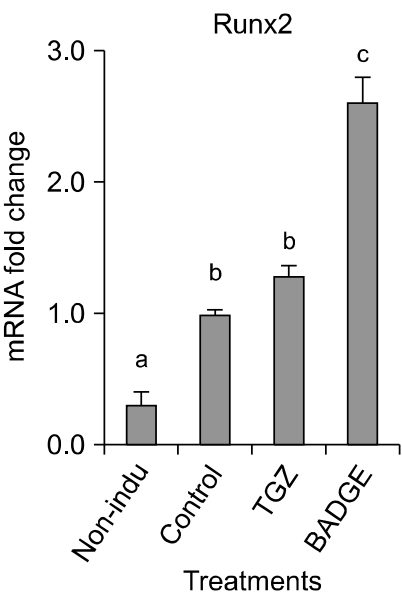

Col I

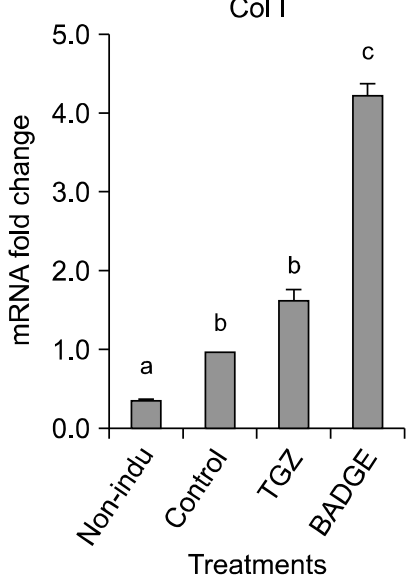

Fig. 4. Gene expression of osteoblast and adipocyte markers in osteogenic-induced porcine skin-derived stem cells treated with troglitazone (TGZ) or bisphenol A diglycidyl ether (BADGE). Levels of adipogenic and osteogenic-related genes were assayed by quantitative real-time polymerase chain reaction. Gene expression was normalized to glyceraldehyde-3-phosphate dehydrogenase $(\mathrm{GAPDH})$ and presented as fold-change from osteogenic-induced control. All data are expressed as relative activity compared to osteogenic-induced control (mean $\pm \mathrm{SE}$ ) from three replicates. Non-indu, non-induction; Control, induced control; PPAR 2 , peroxisome proliferator-activated receptor gamma 2; aP2, adipocyte protein 2; C/EBP- $\alpha$, CCAAT/enhancer-binding protein-alpha; Runx2, runt-related transcription factor 2; ALP, alkaline phosphatase; SPP1, osteopontin; Col I, collagen type I. ${ }^{\text {a-d }}$ Values with different letters differ significantly $(p<0.05)$. 

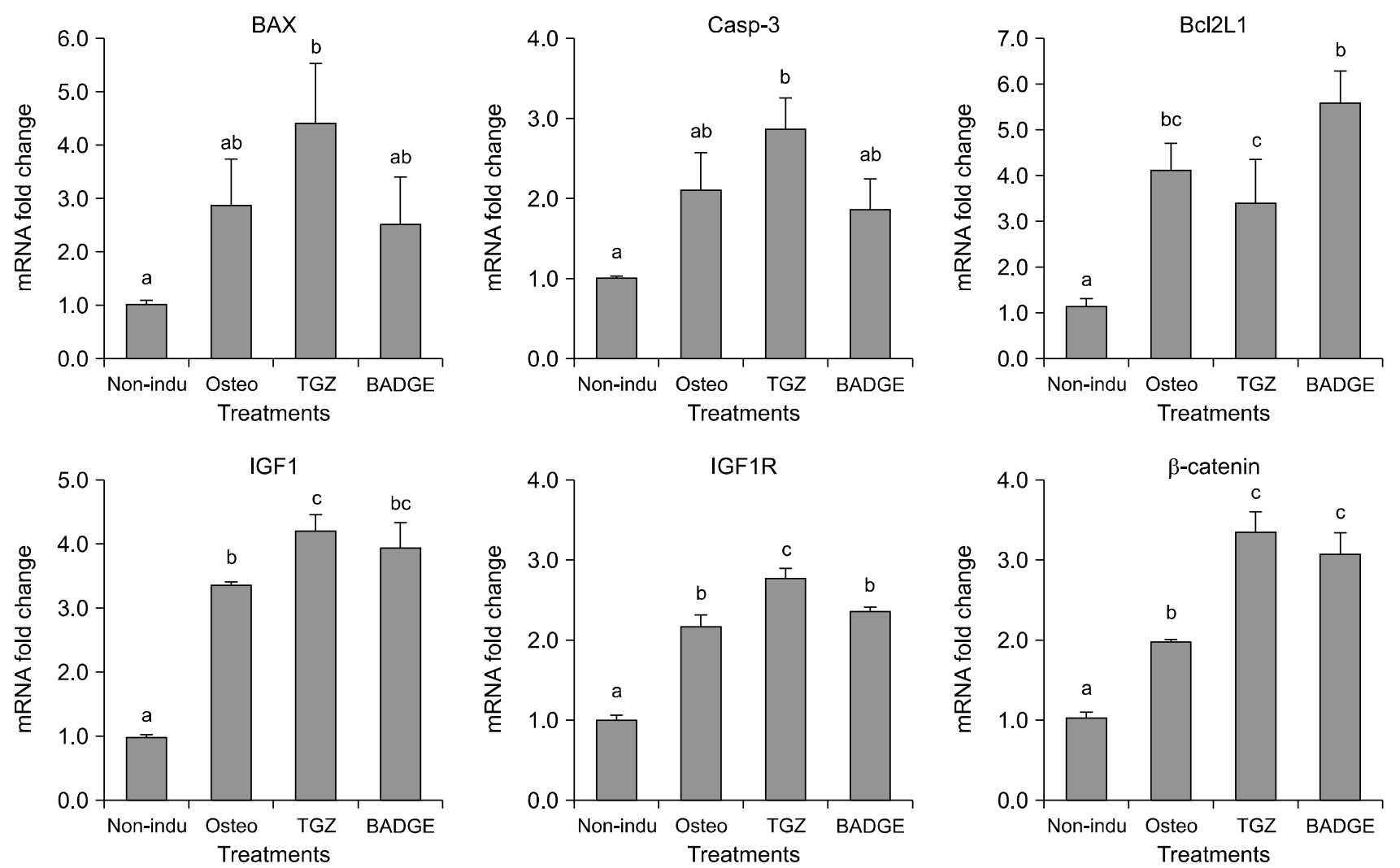

Fig. 5. Expression of apoptotic- and key signaling pathways-related genes controlling both adipogenesis and osteogenesis in porcine skin-derived stem cells (pSSCs) treated with troglitazone (TGZ) or bisphenol A diglycidyl ether (BADGE). Apoptosis and key signaling pathways-related gene levels were assayed by quantitative real-time polymerase chain reaction. Gene expression was normalized to glyceraldehyde-3-phosphate dehydrogenase (GAPDH) and presented as fold-change from non-induced control. All data are expressed as relative activity compared to non-induced control (mean $\pm \mathrm{SE}$ ) from three replicates. These pSSCs were cultured in osteogenic medium with $0.1 \mu \mathrm{M}$ TGZ or $50 \mu \mathrm{M}$ BADGE for 24 days. Non-indu, non-induction; Osteo, osteogenic induction; BAX, BCL2-associated X protein; Casp-3, Caspase-3; BCl2L1, BCL2 like 1; IGF1, insulin-like growth factor 1; IGF1-R, IGF1-receptor. ${ }^{\text {a-c }}$ Values with different letters differ significantly $(p<0.05)$.

treatment but decreased by BADGE treatment (both $p<0.05$ ). The aP2 and C/EBP- $\alpha$ mRNA, as other adipocyte-specific marker genes, showed expression patterns similar to that of PPAR $\gamma 2$ mRNA. Expression levels of osteogenic-related genes, Runx2, ALP, and Col I mRNAs in TGZ-treated pSSCs were slightly higher than those of the control. However, there were no significant differences in expression levels of these genes between TGZ-treated pSSCs and the control except SPP1. BADGE increased expression levels of all four osteogenic-related genes tested in this study: Runx2, ALP, SPP1, and Col I (all $p<$ $0.05)$.

\section{Expression of apoptosis-related genes and critical transcription factors in both adipogenesis and osteogenesis}

Expression levels of BAX and Casp-3 were increased in pSSCs treated with TGZ (Fig. 5). However, they were not significantly different from those in other induced groups. The expression level of BCL2L1, an anti-apoptotic gene, was significantly higher in BADGE-treated pSSCs than that in TGZ-treated ones $(p<0.05)$. Expression levels of nuclear transcription factors that could simultaneously activate adipogenic- and osteogenic differentiation including IGF1, IGF1R, and $\beta$-catenin were significantly higher in pSSCs treated with TGZ than in induced controls $(p<0.05)$.

\section{Discussion}

In this study, we compared the osteogenic differentiation potential of four individual pSSC lines showing varied levels of adipocyte formation during differentiation induction. Our previous study has shown that spontaneous adipocyte generation during chondrogenic induction of pSSCs has a positive effect on chondrogenic differentiation [3]. Different from chondrogenesis, osteogenic differentiation correlated negatively with adipocyte generation during osteogenic induction culture. This result suggests that either adipocyte 
generation restricts osteogenic differentiation or osteogenic differentiation might be restricted in cell lines with strong adipogenic properties.

In the qRT-PCR analysis of osteogenic-induced pSSCs, osteogenic factors, especially Runx 2 and ALP, tended to have expression levels reversely related to expression of PPAR $\gamma 2$ and aP2 mRNAs as adipocyte-specific markers. This suggests that, different from expression of other genes that can be affected by differentiation environments, expressions of Runx 2 and ALP mRNAs are directly regulated by transcriptional factor PPAR $\gamma 2$. PPAR $\gamma$ activity can interfere with the initiation of osteogenic differentiation of MSCs by inducing adipocyte differentiation [1,29]. Different expression patterns among four osteocyte-specific makers might be due to the different operating period of each marker. PPAR $\gamma 2$ and Runx 2 mRNAs are mainly expressed in early stages while aP2, Col I, and SPP1 $\mathrm{mRNAs}$ are expressed in late stages of adipogenic or osteogenic differentiation [13,32]. Distinct gene expression has also been observed during proliferation, extracellular matrix synthesis, and maturation of multipotential progenitor cells in osteogenesis [32]. Interestingly, Runx 2 mRNA expression and calcium accumulation contents from pSSCs-II had the highest levels in this study. This suggests that Runx2 can modulate late mineralization vitally such as calcification of bone and early stage of osteogenic differentiation processes both in vivo and in vitro [29]. However, the expression pattern of representative genes during differentiation of pSSCs into a specific lineage is currently unclear.

Many studies have demonstrated that bone formation and adipogenesis are closely related to PPAR $\gamma[4,11]$. Several studies on PPAR $\gamma$ modulators have been conducted to define the mechanism of cell differentiation $[9,16,19]$. An in vitro study using primary rat osteoblasts treated with TGZ and RGZ (commonly used to treat hyperlipidemia and type 2 diabetes) exhibited lipid droplet formation accompanied by increased expression of PPAR $\gamma 2$ at the expense of osteoblast formation [19]. In this study, ORO extraction level and expression levels of PPAR $\gamma 2$, aP2, and C/EBP- $\alpha$ mRNAs were increased by TGZ treatment. However, calcium content of osteogenic-induced pSSCs was slightly increased rather than decreased. This was supported by the osteogenic gene expression results. Many studies have reported that antidiabetic drugs such as TZD (also known as glitazones) can control lipid homeostasis and adipogenic differentiation by binding to PPAR $\gamma$ receptors, although it can also increase $\mathrm{Mg}^{2+}$ concentration and limit intracellular and extracellular action of $\mathrm{Ca}^{2+}[17,23]$. It has been reported that TGZ blocks $\mathrm{Ca}^{2+}$ release from intracellular stores by increasing the activity of PPAR $\gamma$ receptor in cells $[21,23]$. Blocking intracellular $\mathrm{Ca}^{2+}$ release might be responsible for the relatively high calcium content and expression levels of osteogenic genes in TGZ-treated pSSCs. Bruedigam et al. [6] have reported that RGZ-mediated activation and overexpression of PPAR $\gamma$ can accelerate osteogenic and adipogenic differentiation. $\mathrm{Yu}$ et al. [27] have suggested that PPAR $\gamma$ mRNA expression during osteogenesis has no inevitable negative effect on osteogenic induction.

On the other hand, it has been reported that the use of PPAR $\gamma$ inhibitors can enhance the capacity of human MSCs (hMSCs) for osteogenic cytotherapy [12]. Treatment of hMSCs with GW9662, an inhibitor of PPAR $\gamma$ mRNA, can enhance early osteoblast-specific markers ALP and osteoprotegerin and increase the ability of cells to repair bone lesions both in vitro and in vivo [12]. Thus, PPAR $\gamma$ transcriptional activities could be regarded as having anti-osteoblastic or pro-adipocytic properties. BADGE acts as an antagonist for PPAR $\gamma$. It can abolish the protective effect of a PPAR- $\gamma$ agonist [5]. In murine models, BADGE inhibited adipocyte formation in bone marrow stromal progenitors and promoted hematopoietic recovery [30]. Our results showed that BADGE treatment during osteogenic induction culture of pSSCs reduced lipid droplet formation, blocked PPAR $\gamma 2$ and aP2 transcriptional activities, and increased cell calcium content and expression levels of osteoblast-specific genes, thus having a positive effect on osteogenesis. Meanwhile, it has been reported that a lentivirus-mediated knockdown of PPAR $\gamma$ can inhibit adipogenesis without promoting osteogenesis of hMSCs [27]. Yu et al. [27] suggested that inhibition of PPAR $\gamma$ mRNA expression during osteogenesis does not necessarily result in enhanced osteogenic capability of MSCs. Furthermore, one study has suggested that the agonist effects of BADGE on $\operatorname{PPAR} \gamma$, adipogenesis, and other cellular processes might be cell-type specific [7].

In the present study, osteoclast differentiation was induced well despite the induction of adipocytes by TGZ treatment due to the high expression of nuclear transcription factors promoting both adipogenesis and osteogenesis such as IGF1, IGF1R, and $\beta$-catenin mRNAs (Fig. 5). Control of simultaneous adipogenic and osteogenic differentiation of MSCs has been conducted for both bone morphogenic protein and IGF1 signaling [11]. These two signaling pathways induce adipogenesis and osteogenesis. IGF1 is known as the most abundant growth factor in the bone matrix. It is regulated by growth hormone and can stimulate osteogenic differentiation of MSC by activating mammalian target of rapamycin (mTOR) through the PI3K-Akt pathway [26]. Wabitsch et al. [25] reported that IGF1 can induce critical cell division of adipocyte precursor cells.

Apoptosis has a critical role in bone generation, development, and maintenance through bone remodeling processes such as resorption of osteoblasts. In this study, there was no significant difference in the relative expression levels of pro-apoptosis-related genes such as BAX and Casp-3 mRNAs between the TGZ and BADGE treatment groups. However, the expression of BCL2L1, an anti-apoptotic gene, was significantly higher in 
BADGE-treated pSSCs than in TGZ-treated ones. It has been reported that overexpression of BCL2L1 in osteoblasts can induce apoptosis and inhibit osteoblast differentiation. However, relative expression levels of Col Ia1, SPP1, and osteocalcin (osteoblast-specific genes) in transgenic mice with low expression of BCL2L1 were slightly higher than those in wild type [15]. In addition, it has been reported that osteoblast adhesion and differentiation are promoted in vitro in BCL2L1 transgenic mice, different from results in vivo [28]. There is still debate about how BCL2L1 expression affects osteogenic differentiation or bone development. However, it is clear that apoptosis-induced bone resorption can lead to initiation signaling of progenitor cells or osteogenic differentiation for bone remodeling [22].

In conclusion, we have demonstrated that treatment with BADGE, as a PPAR $\gamma 2$ antagonist, could improve osteogenic differentiation of pSSCs, resulting in remarkable increases of calcium content and expression of osteoblast-specific marker genes. Results of this study show that spontaneous adipocyte generation does not adversely affect osteogenic differentiation. However, reducing spontaneous adipocyte generation by inhibiting PPAR $\gamma 2$ mRNA expression can enhance in vitro osteogenic differentiation of pSSCs.

\section{Acknowledgments}

This study was supported by Basic Science Research Program through the National Research Foundation of Korea (NRF) funded by the Ministry of Education (NRF-2013R1A1A2006182).

\section{Conflict of Interest}

The authors declare no conflicts of interest.

\section{References}

1. Akune T, Ohba S, Kamekura S, Yamaguchi M, Chung UI, Kubota N, Terauchi Y, Harada Y, Azuma Y, Nakamura K, Kadowaki T, Kawaguchi H. PPAR $\gamma$ insufficiency enhances osteogenesis through osteoblast formation from bone marrow progenitors. J Clin Invest 2004, 113, 846-855.

2. Aubert RE, Herrera V, Chen W, Haffner SM, Pendergrass $M$. Rosiglitazone and pioglitazone increase fracture risk in women and men with type 2 diabetes. Diabetes Obes Metab 2010, 12, 716-721.

3. Bae HK, Jung BD, Lee S, Park CK, Yang BK, Cheong HT. Chondrogenic differentiation of porcine skin-derived stem cells with different characteristics of spontaneous adipocyte formation. J Embryo Trans 2017, 32, 193-200.

4. Beresford JN, Bennett JH, Devlin C, Leboy PS, Owen ME. Evidence for an inverse relationship between the differentiation of adipocytic and osteogenic cells in rat marrow stromal cell cultures. J Cell Sci 1992, 102, 341-351.
5. Botolin S, McCabe LR. Inhibition of PPAR $\gamma$ prevents type I diabetic bone marrow adiposity but not bone loss. J Cell Physiol 2006, 209, 967-976.

6. Bruedigam C, Eijken M, Koedam M, van de Peppel J, Drabek K, Chiba H, van Leeuwen JP. A new concept underlying stem cell lineage skewing that explains the detrimental effects of thiazolidinediones on bone. Stem Cells 2010, 28, 916-927.

7. Chamorro-García R, Kirchner S, Li X, Janesick A, Casey SC, Chow C, Blumberg B. Bisphenol A diglycidyl ether induces adipogenic differentiation of multipotent stromal stem cells through a peroxisome proliferator-activated receptor gamma-independent mechanism. Environ Health Perspect 2012, 120, 984-989.

8. Gimble JM, Robinson CE, Wu X, Kelly KA. The function of adipocytes in the bone marrow stroma: an update. Bone 1996, 19, 421-428.

9. Hong KM, Belperio JA, Keane MP, Burdick MD, Strieter RM. Differentiation of human circulating fibrocytes as mediated by transforming growth factor- $\beta$ and peroxisome proliferator-activated receptor $\gamma$. J Biol Chem 2007, 282, 22910-22920.

10. Hwang IS, Bae HK, Cheong HT. Comparison of the characteristics and multipotential and in vivo cartilage formation capabilities between porcine adipose-derived stem cells and porcine skin-derived stem cell-like cells. Am J Vet Res 2015, 76, 814-821.

11. James AW. Review of signaling pathways governing MSC osteogenic and adipogenic differentiation. Scientifica (Cairo) 2013, 2013, 684736.

12. Krause U, Haris S, Green A, Ylostalo J, Zeitouni S, Lee N, Gregory CA. Pharmaceutical modulation of canonical Wnt signaling in multipotent stromal cells for improved osteoinductive therapy. Proc Natl Acad Sci U S A 2010, 107, 4147-4152.

13. Li X, Cui Q, Kao C, Wang GJ, Balian G. Lovastatin inhibits adipogenic and stimulates osteogenic differentiation by suppressing PPAR $\gamma 2$ and increasing Cbfa1/Runx 2 expression in bone marrow mesenchymal cell cultures. Bone 2003, 33, 652-659.

14. Meier C, Kraenzlin ME, Bodmer M, Jick SS, Jick H, Meier CR. Use of thiazolidinediones and fracture risk. Arch Intern Med 2008, 168, 820-825.

15. Moriishi T, Maruyama Z, Fukuyama R, Ito M, Miyazaki T, Kitaura H, Ohnishi H, Furuichi T, Kawai Y, Masuyama R, Komori H, Takada K, Kawaguchi H, Komori T. Overexpression of $\mathrm{Bcl} 2$ in osteoblasts inhibits osteoblast differentiation and induces osteocyte apoptosis. PLoS One 2011, 6, e27487.

16. Muruganandan S, Roman AA, Sinal CJ. Adipocyte differentiation of bone marrow-derived mesenchymal stem cells: cross talk with the osteoblastogenic program. Cell Mol Life Sci 2009, 66, 236-253.

17. Nadler J, Scott S. Evidence that pioglitazone increases intracellular free magnesium concentration in freshly isolated rat adipocytes. Biochem Biophys Res Commun 1994, 202, 416-421.

18. Oshima K, Nampei A, Matsuda M, Iwaki M, Fukuhara A, Hashimoto J, Yoshikawa H, Shimomura I. Adiponectin 
increases bone mass by suppressing osteoclast and activating osteoblast. Biochem Biophys Res Commun 2005, 331, 520-526.

19. Patel JJ, Butters OR, Amett TR. PPAR agonists stimulate adipogenesis at the expense of osteoblast differentiation while inhibiting osteoclast formation and activity. Cell Biochem Funct 2014, 32, 368-377.

20. Pittenger MF, Mackay AM, Beck SC, Jaiswal RK, Douglas R, Mosca JD, Moorman MA, Simonetti DW, Craig S, Marshak DR. Multilineage potential of adult human mesenchymal stem cells. Science 1999, 284, 143-147.

21. Reinhart RA. Magnesium metabolism. A review with special reference to the relationship between intracellular content and serum levels. Arch Intern Med 1988, 148, 2415-2420.

22. Rochefort GY, Pallu S, Benhamou CL. Osteocyte: the unrecognized side of bone tissue. Osteoporos Int 2010, 21, $1457-1469$

23. Takaya J, Higashino $\mathbf{H}$, Kobayashi Y. Intracellular magnesium and insulin resistance. Magnes Res 2004, 17, 126-136.

24. Tchoukalova YD, Hausman DB, Dean RG, Hausman GJ. Enhancing effect of troglitazone on porcine adipocyte differentiation in primary culture: a comparison with dexamethasone. Obes Res 2000, 8, 664-672.

25. Wabitsch M, Hauner H, Heinze E, Teller WM. The role of growth hormone/insulin-like growth factors in adipocyte differentiation. Metabolism 1995, 44 (10 Suppl 4), 45-49.

26. Xian L, Wu X, Pang L, Lou M, Rosen CJ, Qiu T, Crane J,
Frassica F, Zhang L, Rodriguez JP, Jia X, Yakar S, Xuan S, Efstratiadis A, Wan M, Cao X. Matrix IGF-1 maintains bone mass by activation of mTOR in mesenchymal stem cells. Nat Med 2012, 18, 1095-1101.

27. Yu WH, Li FG, Chen XY, Li JT, Wu YH, Huang LH, Wang Z, Li P, Wang T, Lahn BT, Xiang AP. PPAR $\gamma$ suppression inhibits adipogenesis but does not promote osteogenesis of human mesenchymal stem cells. Int J Biochem Cell Biol 2012, 44, 377-384.

28. Zhang W, Pantschenko AG, McCarthy MB, Gronowicz G. Bone-targeted overexpression of Bcl-2 increases osteoblast adhesion and differentiation and inhibits mineralization in vitro. Calcif Tissue Int 2007, 80, 111-122.

29. Zhang X, Yang M, Lin L, Chen P, Ma KT, Zhou CY, Ao YF. Runx2 overexpression enhances osteoblastic differentiation and mineralization in adipose--derived stem cells in vitro and in vivo. Calcif Tissue Int 2006, 79, 169-178.

30. Zhu RJ, Wu MQ, Li ZJ, Zhang Y, Liu KY. Hematopoietic recovery following chemotherapy is improved by BADGE-induced inhibition of adipogenesis. Int J Hematol 2013, 97, 58-72.

31. Zhuang H, Zhang X, Zhu C, Tang X, Yu F, Shang GW, Cai X. Molecular mechanisms of PPAR- $\gamma$ governing MSC osteogenic and adipogenic differentiation. Curr Stem Cell Res Ther 2016, 11, 255-264.

32. Zou L, Zou X, Chen L, Li H, Mygind T, Kassem M, Bünger C. Multilineage differentiation of porcine bone marrow stromal cells associated with specific gene expression pattern. J Orthop Res 2008, 26, 56-64. 